\title{
ANALYSIS AND SIMULATION OF PROGRESSIVE DIE
}

\author{
Raja Shakarappa \\ Assistant Professor, Dept. of Mechanical Engineering, BKIT, Bhalki
}

\begin{abstract}
The aim is to reduce the cost of the progressive dies without compromising on the quality of output. Using the optimum resources possible in designing the progressive dies frame can effect this reduction in the cost of the progressive dies. One way of doing it will be optimizing the volume of material utilized for building the structure. An attempt has been made in this direction to reduce the volume of material. In this project work an industrial application project consisting of mass minimization of the progressive dies is considered. This progressive dies has to compensate the stresses acting on the tools, top, middle \& bottom plates and to fulfill certain critical constraints. The deformation of the frame with constant thickness is described by the plain stress state equation for linear elasticity. COSMOS is the commercial software that has been used for this analysis which uses finite element method for solution. The methodology followed in this work is comparison of stresses induced in the machine for different thickness used construction of frame of the progressive dies. These stresses are compared to yield stress and considering minimum factor of safety 2.0, the thickness of frame of the progressive dies selected to reduce the volume of material utilized for building the structure and hence to reduce the cost of the machine.
\end{abstract}

Keywords: Production, Dies, Tools, Production Time.

\section{INTRODUCTION}

Progressive die can perform many operation compared to other dies and also it can able to eliminate the loading \& unloading time which results in faster production rate.

\subsection{What is a Progressive Die?}

Progressive die is a kind of assembled die where in more than one operation can be done at a time. This progressive die is applicable mainly on sheet metal operations, where in all the tools are previously loaded in a sequence as per the operation requirements.

\subsection{What is Analysis of Progressive Die?}

Here in our project we are concentrating over structural analysis of progressive dies in which it includes the following.

- Materials used to produce progressive die.

- Materials used to produce tools in progressive die.

- What will be the sequence of progressive die tools?

- What are the thermal effects on tools \& die.

- What will be the changes to be done in progressive die to improve productivity, etc...

\subsection{Types of Sheet Metal Works}

\subsubsection{Blanking}

It is a process in which the punch removes a portion of metal from the stock of sheet metal of the necessary thickness \& width.

\subsubsection{Piercing}

It is also sometimes known as punching, the piercing is making holes in a sheet.

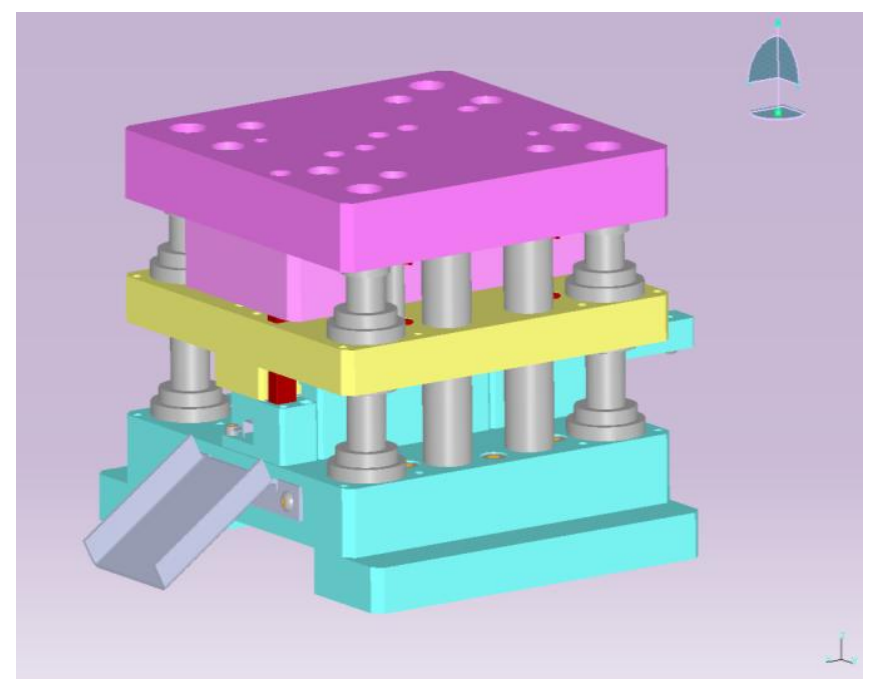

Fig 1.1 progressive die assembled view

\subsubsection{Trimming}

In operations such as drop forging die casting, a small amount of extra metal gets spread out near parting planes which is known as flash. The flash is to be trimmed before the castings or forging is to be used. 


\subsubsection{Shaving}

In blanking or piercing operation, the edge of the blank or the hole is not perfectly clean because of the burr generated in the sheering process, which is equal to the clearance on the die. For close tolerance work, the blanking or piercing process is followed by shaving which removes the burr left on the product.

\subsubsection{Nibbling}

Nibbling is removing the metal in small increment, it is when a specific counter is to be cut in a sheet metal, and a small punch is used to punch repeatedly along the necessary contour, generating the required profile.

\subsubsection{Notching}

Notching is a method to cut a specified small portion of metal toward the edge of stock.

\subsubsection{Bending}

It is the operation where in a sheet metal is bent over a die by the help of stress.

\subsubsection{Embossing}

Embossing is the operation used in making raised figures on sheet with its corresponding relief on the other side.

\subsubsection{Coining}

Coining is essentially a cold forging operation except for the fact that the flow of metal occurs only at the top layer \& not the entire volume.

\subsubsection{Spinning}

Spinning is the process used for making cup shaped articles which are axisymmetrical. The process of spinning consists of rotating the blank, fixed against the form block \& then applying gradually moving forces so that, the blank takes the shapes of the form blocks.

\subsection{Objectives}

Since progressive dies initial implementation cost is very high, so by analyzing it thermally \& structurally decision making of implementation of this die becomes easy. By the analysis of progressive die we can suggests the changes that can be done in tooling material and the die itself to improve the productivity. This can be done by knowing the stress acting on each part of die as well as on tools which are used in die. Another main objective of this project is we can also calculate increase in productivity in progressive die than normal compound die. This can be done by comparing the time taken in operation of a sheet metal between progressive die and normal die.

\subsection{Advantages and Disadvantages of Progressive} Dies.

\subsubsection{Advantages of Progressive Die}

- $\quad$ Progressive die can operate more than operation at a time in sequence which eliminates the idle time in a sheet metal working.

- Progressive die can produce parts in batch as well as in mass which increases the productivity by large value compared to normal die.

- Progressive dies reduces the man power by operating in continuous sequence.

- In progressive dies quality parts are produced continuously.

- It also eliminates the idle time, loading \& unloading time in operation.

\subsubsection{Disadvantages of Progressive Die}

- Since progressive dies initial cost is very high it rarely used in small scale industries.

- Since progressive dies produce parts in batch as well as in mass production it is not used variable production.

- Even though it reduces manpower still it need a program and a supervisor.

\subsection{Applications}

- Progressive dies are mainly used in automobiles.

- In domestic appliance progressive dies are used.

- In aircrafts also progressive dies are effectively used. In many commercial products progressive dies are used.

- In boilers and some storing devices progressive dies are used.

- In many electronic products progressive dies are used.

- In case of some security devices also progressive dies are used.

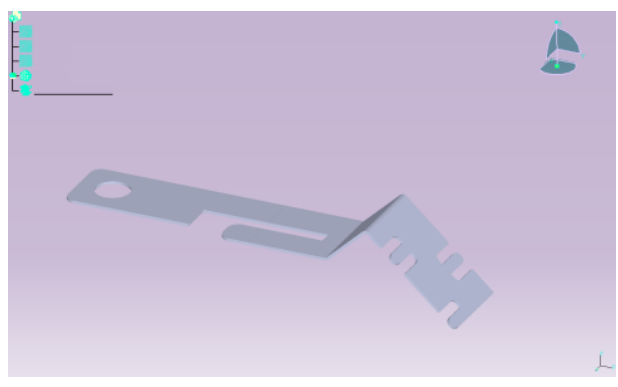

Fig 1.2 final product of progressive die 


\section{NUMERICAL METHODS IN ENGINEERING}

\section{ANALYSIS}

\subsection{Classical Methods}

Classical method attempt to solve field problem directly by forming governing differential equations based on fundamental principles of physics.

\subsubsection{Exact Solution}

Those having closed forms are possible only for the simplest cases of geometry, loading, and boundary conditions. A somewhat wider variety of classical problems can be solved using approximate solutions to the governing differential equations. These solutions take the form of series expansions, which are truncated after a reasonable degree of convergence.

\subsubsection{Approximate Solution}

These solutions require regular geometric shapes, simple boundary condition, and well-behaved loads. Consequently, these solutions bear little resemblance to most practical engineering problems. The principal advantage of classical methods is the high degree of problem insight provided by solutions of this type.

\subsection{Numerical Methods}

Numerical methods address a broad range of problems.

\subsubsection{Energy Method}

This method seeks to minimize an expression for the potential energy of a structure over its entire domain.

\subsubsection{Boundary Elements}

This method approximates functions satisfying the governing differential equations, but not the boundary conditions. Problem size is reduced because Elements represent only the boundary of the domain. However, the application of this method relies on knowing the fundamental solution to the governing equations, which can be difficult to obtain.

\subsubsection{Finite Difference Method}

This method replaces governing differential equations and boundary conditions with corresponding algebraic equations. This permits the representation of somewhat irregular problems, but complex geometry, boundary conditions, or loads become difficult to handle.

\subsubsection{Finite Element Method}

The finite method offers virtually unlimited problem generality by permitting the use of elements of various regular shapes along with wide range of loads and constraints applications. Changing any significant aspect of the model generally requires a complete reanalysis of the problem. Analysts consider this a small price to pay, however, since the finite element method is often the only the possible method of analysis.

\subsection{Finite Element Approach}

\subsubsection{Introduction}

The FEA is a numerical procedure for analyzing structures of complicated shapes, which otherwise would be difficult by classical analytical methods. Analytical solution is a mathematical expression that gives values of desired unknown quantity at any location in a body or a structure and it is valid for an infinite number of locations in body or structure. But analytical solutions can be obtained only for simple engineering problems. It is extremely difficult and many a times impossible to obtain exact analytical mathematical solutions for complex Engg. Problems. In such cases FEM is used which gives approximate solution.

\subsubsection{Description of Method}

The limitations of the human mind are such that it cannot grasp the behavior of its complex surroundings and creations in one opiate finite method offers virtually unlimited problem generality by permitting the use of elements of various regular shapes along with wide range of loads and constraints applications. Changing any significant aspect of the model generally requires a complete reanalysis of the problem. Analysts consider this a small price to pay, however, since the finite element method is often the only the possible method of analysis.

The FEA is a numerical procedure for analyzing structures of complicated shapes, which otherwise would be difficult by classical analytical methods. Analytical solution is a mathematical expression that gives values of desired unknown quantity at any location in a body or a structure and it is valid for an infinite number of locations in body or structure. But analytical solutions can be obtained only for simple engineering problems. It is extremely difficult and many a times impossible to obtain exact analytical mathematical solutions for complex Engg. Problems. In such cases FEM is used which gives approximate solution

The libration. Thus the process of subdividing all systems into their individual components or ,elements "e, whose behavior is readily understood, and then rebuilding the original system from such components to study the behavior is a natural way in which the engineers and scientists proceeds. The finite element method is a powerful tool for the numerical procedure to obtain solutions to many of the problems encountered in engineering analysis. From structural, thermal and heat transfer, fluid dynamics, fatigue related problems, electric and magnetic fields, the concepts of finite element methods can be utilized to these engineering problems. In this method, the 
domain over which the analysis is studied is divided into a number of finite elements. Interpolating functions are used to reduce the behavior at an infinite field of points to a finite number of points. These points define the finite elements. The elements are interconnected by nodes. All the elements are assembled together and the requirements of continuity and equilibrium are to be satisfied between neighboring elements. A unique solution can be obtained to the overall system of linear algebraic equations, provided the boundary conditions of the actual system are satisfied. Solution of these equations gives us the approximate behavior of the continuum. To obtain an accurate solution in the region of rapidly varying variables, more number of smaller elements must be used.

An analytical solution is a mathematical expression that gives the values of the desired unknown quantity at any location in a body, and as a consequence it is valid for an infinite number of locations in the body. For problems involving complex material prosperities and boundary conditions, the engineers resort to finite element methods that provide approximate, but acceptable, solutions as the problem addressed is too complicated to be solved satisfactorily by the classical analytical methods.

FEA works on the principle of divide and rule, that is, it transforms a physical system having infinite unknowns into small finite elements having finite number of unknowns. The unknowns are called degrees of freedom. Degree of freedom is the number of independent coordinates which must be specified to define all displacements. Instead of solving the problem for the entire body in one operation, the solutions are formulated for each constituent unit and combined to obtain the solution for the entire body i.e. going from part to whole Thus in FEM, body or structure is divided into finite number of smaller units known as elements. This process of dividing the body into finite number of elements is known as discretization. The assemblage of elements then represents original body or structure. Physical problem involves an actual structure subjected to certain loads and constraints. The material properties and the governing relationships are considered over the elements and expressed in terms of unknown values at element corners. An assembly process duly considering the loading and constraints, results in a set of equations. Solution of these equations did by FEM gives us the approximate behavior of the continuum. The elements are considered to be interconnected at finite number of joints which are known as nodes or nodal points. The finite element method has become a powerful tool for the numerical solution of wide range of engineering problems. Application range from deformation and stress analysis of automotive, aircraft, building, and bridge structures to field analysis of heat flux, fluid flow, magnetic flux, seepage and other flow problems. Thus we can define FEA precisely as- "Finite element analysis is a mathematical representation of a physical system comprising of a part / assembly (model), material properties, and applicable boundary conditions \{collectively referred to as preprocessing\}, the solution of that mathematical representation \{solving\}, and the study of the results of that solution \{post processing\}."

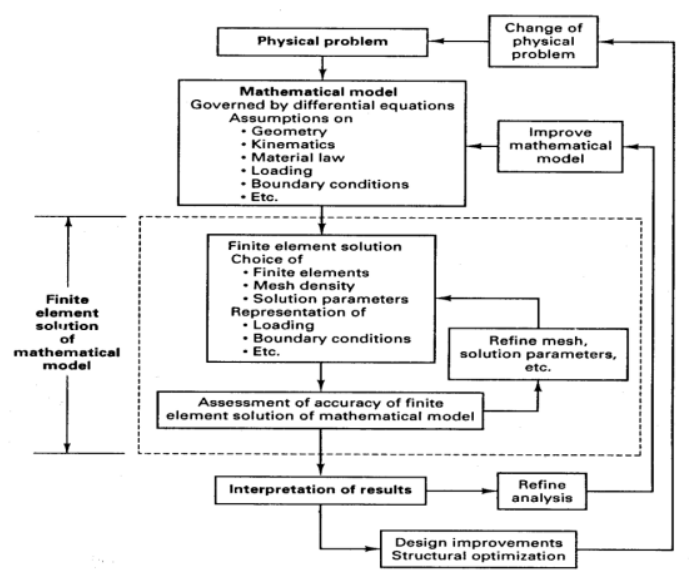

Fig 2.1 Line Diagram Of Analysis

\subsubsection{Advantages and Limitations of FEM}

\subsubsection{Advantages}

FEM permits greater flexibility in considering continuum of complex shapes.

FEM not only accommodates complex geometry and boundary conditions but also proven successful in representing various types of complicated material properties that are difficult to incorporate into other numerical methods.

FEM readily accounts for non-homogeneity by a simple tactic of assigning different properties to different materials.

The symmetric generality of FEM makes it a power full and versatile tool for a wide range of problems. As a result flexible general-purpose computer programs can be constructed.

The FEM programs developed for one field of engineering can be employed for other fields of engineering with little or no modifications.

Though the solution obtained by FEM is discrete node points, it can be extended to all location of the body.

FEM can be employed as a powerful design analysis and validation tool to detect any flaws in the system and critical points even before the actual installation of the system

FEM can be employed for non-engineering applications

\subsubsection{Limitations}

A few complex phenomenons are accommodated adequately by FEM at its current stage of development. The accuracy of this method relies mainly on coefficient and material 
properties, which describe the basic phenomenon. Even the most efficient FEM computer codes require a relatively large amount of computer memory and time. Hence this method is limited to those who have access to relatively large and highsped computers. The process of discretization is tedious one and is likely source of error. Though this process can be automated, error free input data cannot be obtained, as it requires intuitive judgment by the analyst. The large volume of solution information generated by FEM will be worthwhile only when its generation and tempered by proper engineering judgment.

\subsection{Steps Involved In FEM}

The different steps involved in the FE formulation are given below:

Step1: Define the element and shape functions. The first step in any FE analysis in to model the geometry. The model is then divided into a number of elements. The type of elements could be depending on the problem like line, beam, plates or shells etc.

Step2: Constitutive equations To derive an equation for the element stiffness, that is, element forces as functions of element displacements, the displacements are differentiated (to obtain the strains), and then substituted in the material law, in other words, the stress-strain relationships.

Step3: Derivation of elemental stiffness matrix The elements are assigned with section and material properties. The element stiffness matrix depends on these properties. The energy method is normally used for the derivation.

Step4: Assemblage of the element stiffness matrix. The next stage is to assemble all the individual elements to form the overall (global structure). This is done by using the compatibility conditions at nodes. The displacement of a particular node must be the same for every element connected to it. The externally applied loads must also be balanced by the forces on the elements at these nodes.

Step5: Apply the boundary conditions and external loads To obtain a unique solution of the problem, some displacement (i.e. boundary conditions) and loading conditions must be prescribed at some of the nodes. This may be of the following forms.

Displacement condition: a zero or nonzero prescribed nodal displacement.

Force condition: a prescribed applied force in a given direction.

These boundary conditions are incorporated into the system of linear algebraic equations, which can then be solved to obtain a unique solution for the displacements at each node.
Step6: Solve the equations for unknowns Standard equation solvers, such as the Gaussian elimination technique, can be used to solve the equations to determine the unknown variables at each node. Since the stiffness matrix is symmetric and sparsely populate, specially adapted solvers that can considerably reduce the computation time are often used in FE code. Some of the solvers are Frontal solver, skyline solver etc.

Step7: Compute other variables After solving the global equations, displacements at all the nodal points are determined. From the displacement values, the element strains can be obtained from the stress-strains relations. In FE formulation only the displacements are the independent variables, that is, forces, strains and stresses are obtained from the displacements.

\subsection{Structural Analysis}

Structural analysis is probably the most common application of the finite element method. The term structural implies not only civil engineering structures such as bridges and buildings, but also naval, aeronautical, and mechanical structures such as ship hulls, aircraft bodies, and machine housing, as well as mechanical components such as pistons, machine parts, and tools. The types of structural analyses are:

\subsubsection{Static Analysis}

Static analysis calculates the effects of steady loading conditions on a structure, while ignoring inertia and damping effects, such as those caused by time-varying loads. A static analysis can, however, include steady inertia loads (such as gravity and rotational velocity), and time-varying loads that can be approximated as static equivalent loads (such as the static equivalent wind and seismic loads commonly defined in many building codes). Static analysis involves both linear and nonlinear analyses. Nonlinearities can include plasticity, stress stiffening, large deflection, large strain, hyper elasticity, contact surfaces, and creep.

\subsubsection{Linear Static Analysis}

In linear analysis, the behavior of the structure is assumed to be completely reversible; that, the body returns to its original unreformed state upon the removal of applied loads and solutions for various load cases can be superimposed. The assumptions in linear analysis are:

- Displacements are assumed to be linearly dependent on the applied load.

- A linear relationship is assumed between stress and strain.

- Changes in geometry due to displacement are assumed to be small and hence ignored.

- Loading sequence is not important and the final state is not affected by the load history. The load is applied in one go with no iterations. 


\subsubsection{Buckling Analysis}

Used to calculate the buckling loads, critical loads at which the structure becomes unstable and to determine the buckling mode shape- the characteristic shape associated with a structure's buckled response.

\subsubsection{Harmonic Analysis}

Any sustained cyclic load with produce a sustained cyclic response (a harmonic response) in a structural system. Harmonic response analysis gives you the ability to predict the sustained dynamic behavior of structures, thus enabling us to verify whether or not the designs will successfully overcome resonance, fatigue, and other harmful effects of forced vibrations.

\subsubsection{Transient Dynamic Analysis}

Transient dynamic analysis also called as time-history analysis, is a technique used to determine the dynamic response of a structure under the action of any general timedependent loads. This type of analysis is used to determine the time-varying displacements, strains, stresses, and forces in structure as it responds to and combination of static, transient, and harmonic loads. The time scale of the loading is such that the inertia or damping effects are considered to be important.

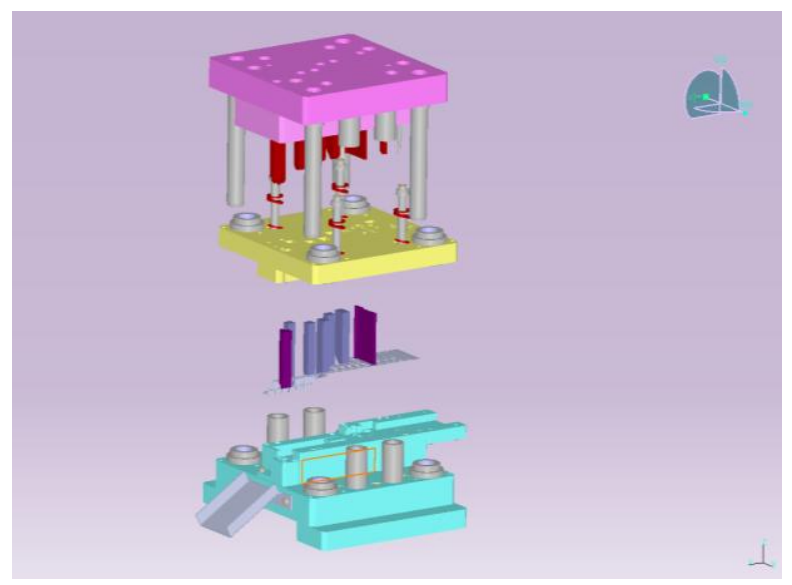

Fig 2.2 Dissembled view of progressive die

\section{COSMOSXPRESS}

COSMOS press offers an easy-to-use first pass stress analysis tool for Solid Works users. COSMOSxpress can help you reduce cost and time-to-market by testing your designs on the computer instead of expensive and time-consuming field tests. For example, you may want to examine the effects of a force applied to the faucet. COSMOSxpress simulates the design cycle and provides stress results. It also shows critical areas and safety levels at various regions in the faucet. Based on these results, you can strengthen unsafe regions and remove material from overdesigned areas. COSMOS press uses the same design analysis technology that COSMOS/Works uses to perform stress analysis. More advanced analysis capabilities are available within the COSMOS/Works line of products. The wizard interface of COSMOSxpress guides you through a five step process to specify material, restraints, and loads, run the analysis, and view the results. The accuracy of the results of the analysis depends on material properties, restraints, and loads. For results to be valid, the specified material properties must accurately represent the part material, and the restraints and loads must accurately represent the part working conditions. COSMOSxpress supports the analysis of solid, single-bodied parts only. It does not support the analysis of assemblies, surface models, or multimode parts.

\subsection{Assigning Material}

The response of the part depends on the material it is made of. COSMOS press must know the elastic properties of the material of your part. You can pick a material from the material library provided with COSMOS press or you can input material properties manually. Materials can be isotropic, orthotropic, or anisotropic. COSMOS press supports isotropic materials only.

To assign material from the material library:

1. On the Material tab, click Define. The Material dialog box appears.

2. Under Select material source, do the following Make sure that Library files are selected.

1. Select the desired material. COSMOS press displays the properties of the selected material in the table to the right.

2. Yield strength (SIGYLD) is not defined for some materials in the material library. SIGYLD is used to calculate factor of safety

3. Under Material model, select Units for displaying the material properties.

4. Click OK. The program assigns the selected material to the part and a check mark appears on the Material tab.

5. Click Next. The Restraint tab appears.

To define material properties:

1. On the Material tab, click Define. The Material dialog box appears.

2. Under Select material source, click Input and type a name for the material.

3. Under Material model, select Units. Make sure to select the unit system before you type the numerical values of the desired properties.

3. In the properties table, enter values under the Value column. You can define the following properties:

- EX (Modulus of elasticity).

- NUXY (Poisson's ratio). If you do not define NUXY, COSMOS press assumes a value of 0 . 
- SIGYLD (Yield Strength). Used only to calculate factors of safety.

- DENS (Mass density). Used to include mass properties of the part in the report file only.

4. Click OK. COSMOS press

Assigns the specified material properties to the part and a check mark appear on the Material tab. 6. Click Next. The Restraint tab appears. Once a material has been assigned to the part, Edit appears instead of Define.

\subsection{Applying Restraints}

On the Restraint tab, you define restraints. Each restraint can contain multiple faces. The restrained faces are constrained in all directions. You must at least restrain one face of the part to avoid analysis failure due to rigid body motion. To apply restraints to the model:

1. Click Next to continue.

2. Type a name for the restraint or accept the default name. We recommend that you use meaningful names for the restraints.

3. In the graphics area, click a face to restrain. To select additional faces, hold down the Ctrl key while you click the faces.

4. Click Show symbol to view the restraint.

5. Click Next. A check mark appears on the Restraint tab and a list of the restraints appears. Click the appropriate button to Add, Edit, or Delete a restraint.

6. Click Next. The Load tab appears.

\subsection{Applying Loads}

On the Load tab, you apply force and pressure loads to faces of the model

\subsubsection{Forces}

You can apply multiple forces to a single face or to multiple faces.

To apply a force load:

1. Click Next to continue.

2. Select Force and click next.

3. Type a name for the force or accept the default name.

4. In the graphics area, click the desired face and click next. To select multiple faces, hold down the Ctrl key while you click the faces.

5. Select: - Normal to each selected face to apply the force in the direction normal to each selected face. Or - Normal to a reference plane to apply the force to a selected reference plane. If you select this option, you need to select a reference plane in the Feature Manager Design tree. 6. Select the force units first, and then type the force value. The specified force value is applied to each face.
Select 3 faces and specify a $50 \mathrm{lb}$ force; COSMOS press applies a total force of $150 \mathrm{lbs}$ (50 lbs on each face).

7. Click Show symbol to make sure that the load is applied in the desired direction. Click Flip direction to reverse the direction of the force.

8. Click Next. The force list box lists the defined force. A check mark appears on the Load tab. Click the appropriate button to Add, Edit, or Delete a force.

9. Click Next. The Analyze tab appears.

\subsubsection{Pressure}

You can apply multiple pressures to a single face or to multiple faces. COSMOSxpress applies pressure loads normal to each face. To apply a pressure:

1. Click Next to continue.

2. Select Pressure and click Next.

3. Type a name for the pressure, or accept the default name.

4. In the graphics area, click the desired face and click Next.

5. Select the pressure units, then type the pressure value.

6. Click Show symbol to make sure that the load is applied in the desired direction. Click Flip direction to reverse the direction of the pressure.

7. Click Next. The pressure list box lists the defined pressure. A check mark appears on the Load tab. Click the appropriate button to Add, Edit, or Delete a pressure.

8. Click Next. The Analyze tab appears.

\subsection{Analyzing the Part}

COSMOS press prepares the model for analysis and then it calculates displacements, strains, and stresses. When the existing results do not belong to the current geometry, material, restraints, or loads, an Update button appears in the lower left corner of the COSMOS press window. Click Update to re-analyze the model and calculate the new results.

To analyze the part:

1. Read the displayed information and click Next.

2. Select: Yes (recommended) to accept the default mesh settings (default element size and tolerance values) or No, I want to change the settings to change the default mesh settings.

3. Click Next. If you choose to change the default settings, type in the desired values, or drag the slider. The default tolerance is $5 \%$ of the specified element size. Click Next. For a more accurate solution, drag the slider towards the right (finer). For a quick estimate, drag the slider to the left (coarser).

4. Click Run.

When analysis is completed, a check mark appears on the Analyze tab, and the Results tab appears. If COSMOS press fails to mesh the part, you will receive a message. Try to analyze again using one or more of the following options: If the part is complex, suppress small fillets and otherandotherfeatures that are not significant for analysis. Use 
a smaller element size of about $80 \%$ of the previous one. Use a larger tolerance of up to $30 \%$ of the element size. The default tolerance is $5 \%$ of the element size. COSMOS/Works provides additional tools to deal with mesh failure. Viewing the Results After completing the analysis, you can view results. A check mark on the Results tab indicates that results exist and are available to view for the current geometry, material, restraints, and loads. When the existing results do not belong to the current geometry, material, restraints, or loads, an Update button appears in the lower left corner of the COSMOS press window. Click Update to re-analyze the model and calculate the new results. Although COSMOS press calculates displacements, strains, and stresses, it only allows you to view stresses. The first screen of the Results tab displays the minimum factor of safety among all locations in the part. Standard engineering codes usually require a factor of safety of 1.5 or larger. For a given minimum factor of safety, COSMOS press plots possible safe and unsafe areas in blue and red, respectively.

\subsubsection{How to Assess the Safety of your Design?}

1. To view regions of the model with a factor of safety less than a given value, type this value in the box and click Show Me. COSMOS press displays regions of the model with factors of safety less than the specified value in red (unsafe regions) and regions with higher factors of safety in blue (safe regions).

2. To view more results, click Next

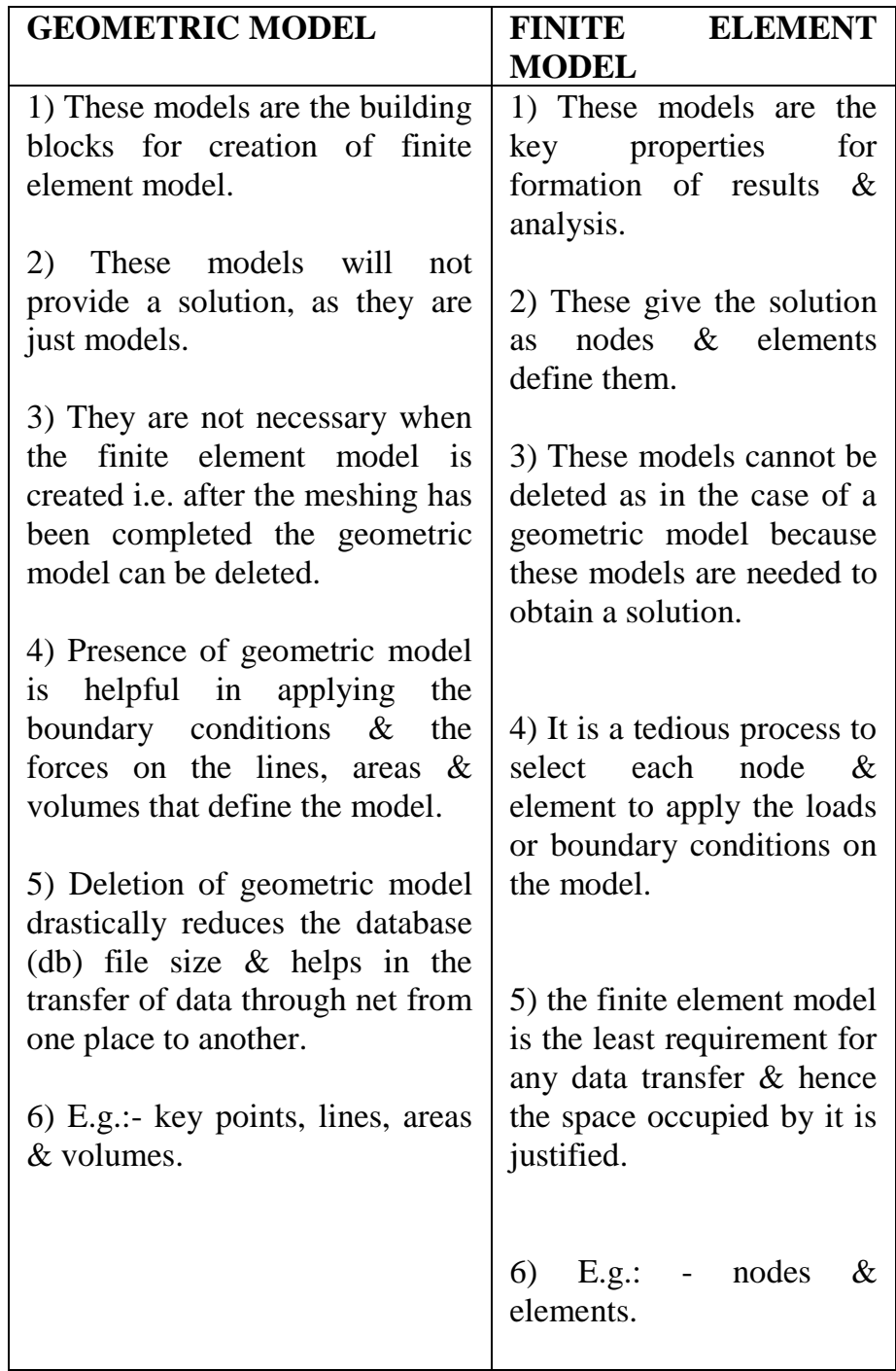

3.4.2 How to View the Stress Distribution in the Model?

1. Click Show me the stress distribution in the model.

2. Click Next. The equivalent stress (von Misses stress) plot is generated.

3. Click any of the following:

- Play to animate the stress plot.

- Stop to stop the animation.

- Save to save the animation to a separate file.

\subsubsection{How to View the Deformed Shape of the Model?}

1. Click Show me the deformed shape of the model.

2. Click Next. The deformed shape plot is generated.

3. Click any of the following:

- Play to animate the deformed shape plot.

- Stop to stop the animation.

- Save to save the animation to a separate file. 


\section{ANALYSES}

\subsection{Analysis OF Progressive Die Parts}

The following is the details for analyzing the 3 parts of progressive dies

4.1.1 Steel, high strength 4340

4.1.2 Modulus of elasticity $=210000000000 \mathrm{n} / \mathrm{m} 2$

4.1.3 Density $=7.8 \mathrm{~g} / \mathrm{cc}$

4.1.4 Poi $=0.29$

4.1.5 Uts $=1550$

4.1.6) pressure $100 \mathrm{kgf} / \mathrm{cm} 2$ tools

4.1.7) plate $200 \mathrm{kn}$

Working stress

4.1.8) top plate $=276 \mathrm{n} / \mathrm{mm} 2$

4.1.9) mid plate $=356 \mathrm{n} / \mathrm{mm} 2$

4.1.10) bottom plate $=183 \mathrm{n} / \mathrm{mm} 2$

\subsubsection{ANALYSIS OF TOP PLATE (max stress=} $\left.2.761 \mathrm{e}^{\wedge} 008\right)\left(\mathrm{N} / \mathrm{m}^{\wedge} 2\right)$

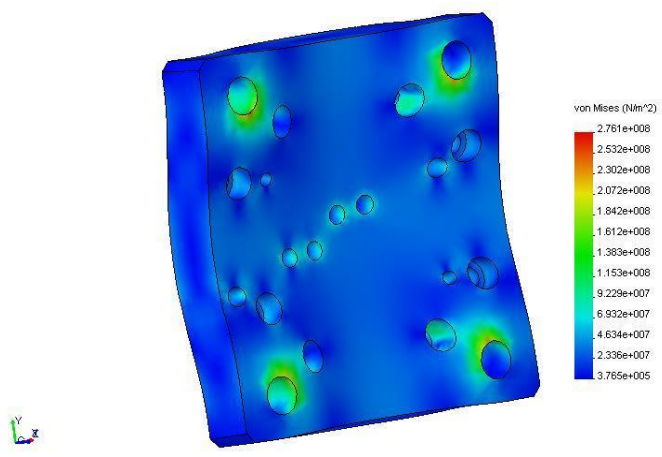

Fig 4.1.1 Top plate

\subsubsection{Analysis of Middle Plate (max stress=} $\left.3.569 \mathrm{e}^{\wedge} 008\right)\left(\mathrm{N} / \mathrm{m}^{\wedge} 2\right)$

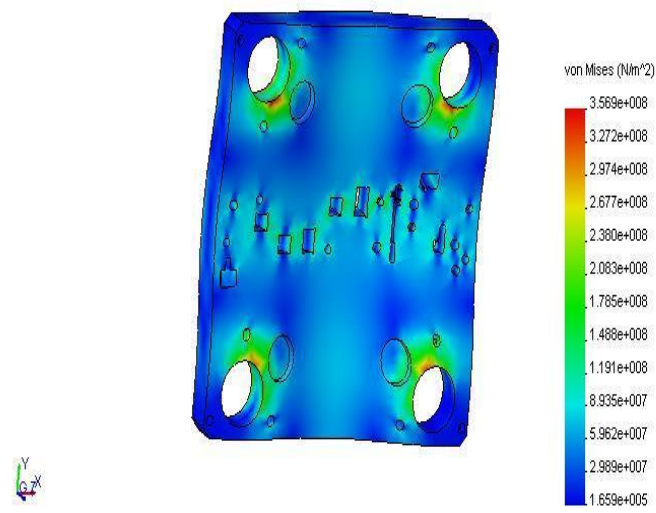

Fig 4.1.2 Middle plate

\subsubsection{Analysis of Bottom Plate (max stress= $\left.1.835 \mathrm{e}^{\wedge} 008\right)\left(\mathrm{N} / \mathrm{m}^{\wedge} 2\right)$}

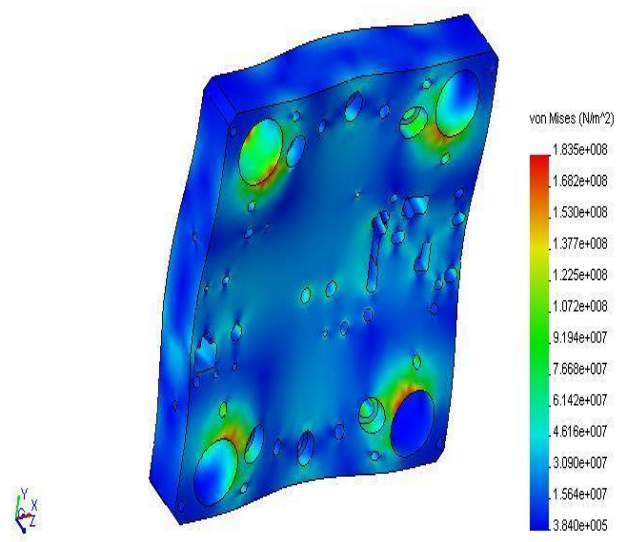

Fig 4.1.3 bottom plate

\subsection{Analysis of Progressive Die Tools}

4.2.1 The Following is the Details for Analyzing the

\section{Tools of Progressive Die}

4.2.1) Steel, high strength 4340

4.2.2) modulus of elasticity $=210000000000 \mathrm{n} / \mathrm{m} 2$

4.2.3) density $=7.8 \mathrm{~g} / \mathrm{cc}$

4.2.4) poi $=0.29$

4.2.5) uts $=1550$

4.2.6) pressure $100 \mathrm{kgf} / \mathrm{cm} 2$ tools

4.2.7) plate $200 \mathrm{kn}$

Working stress

4.2.8) top plate $=276 \mathrm{n} / \mathrm{mm} 2$

4.2.9) mid plate $=356 \mathrm{n} / \mathrm{mm} 2$

4.2.10) bottom plate $=183 \mathrm{n} / \mathrm{mm} 2$

4.2.1 Analysis of forming punch 01 (max stress= $\left.1.345 \mathrm{e}^{\wedge} 008\right)\left(\mathrm{N} / \mathrm{m}^{\wedge} 2\right)$
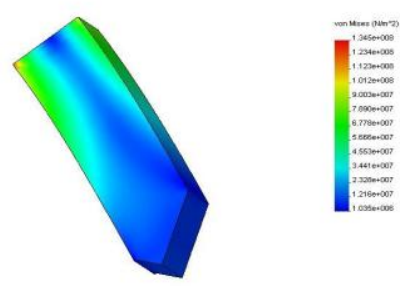

Fig 4.2.1 forming punch 01 
4.2.2 Analysis of Forming Punch 02 (max stress= $\left.1.180 \mathrm{e}^{\wedge} 008\right)\left(\mathrm{N} / \mathrm{m}^{\wedge} 2\right)$

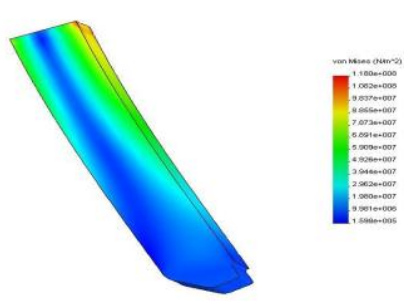

$x^{2}$

Fig 4.2.2 forming punch 02

4.2.3 Analysis of Forming Punch 03 (max stress= $\left.8.744 \mathrm{e}^{\wedge} 007\right)\left(\mathrm{N} / \mathrm{m}^{\wedge} 2\right)$
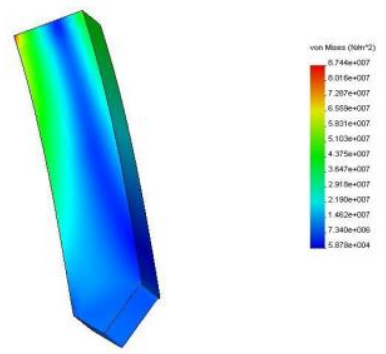

$t^{2}$

Fig 4.2.3 forming punch 03

4.2.4 Analysis of forming punch 04 (max stress= $\left.9.269 \mathrm{e}^{\wedge} 007\right)\left(\mathrm{N} / \mathrm{m}^{\wedge} 2\right)$
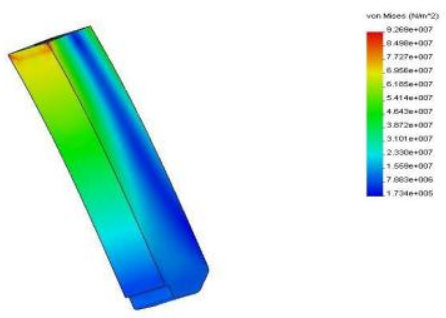

\.

Fig 4.2.4 forming punch 04
4.2.5 Analysis of Forming Punch 05 (max stress= $\left.3.764 \mathrm{e}^{\wedge} 008\right)\left(\mathrm{N} / \mathrm{m}^{\wedge} 2\right)$

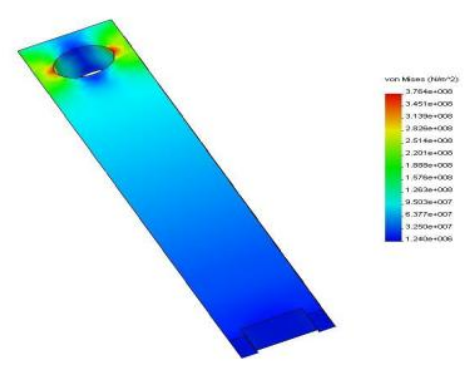

Fig 4.2.5 forming punch 05

4.2.6 Analysis of Forming Punch 06 (max stress= $\left.2.162 \mathrm{e}^{\wedge} 008\right)\left(\mathrm{N} / \mathrm{m}^{\wedge} 2\right)$
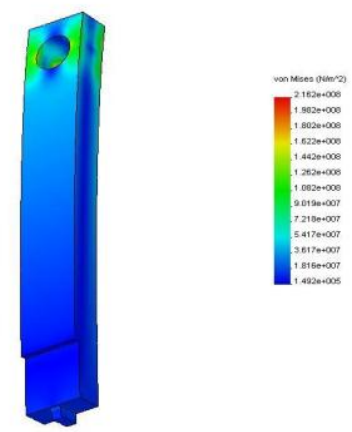

$x^{2}$

Fig 4.2.6 forming punch 06

4.2.7 Analysis of forming punch 07 ( $\max$ stress= 4.270 $\left.\mathrm{e}^{\wedge} 007\right)\left(\mathrm{N} / \mathrm{m}^{\wedge} 2\right)$
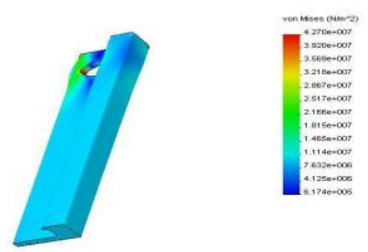

Fig 4.2.7 forming punch 07 
4.2.8 Analysis of Forming Punch 08 (max stress= $\left.1.550 \mathrm{e}^{\wedge} 008\right)\left(\mathrm{N} / \mathrm{m}^{\wedge} 2\right)$

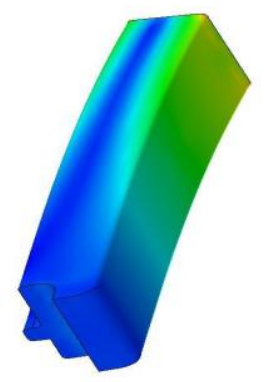

Fig 4.2.8 forming punch 08

4.2.9 Analysis of Forming Punch 09 (max stress= $\left.4.540 \mathrm{e}^{\wedge} 008\right)\left(\mathrm{N} / \mathrm{m}^{\wedge} 2\right)$

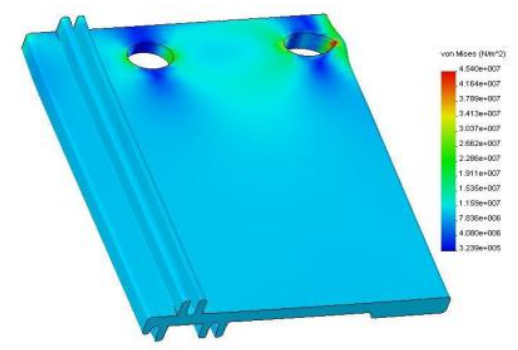

$V^{x}$

Fig 4.2.9 forming punch 09

4.2.10 Analysis of Forming Punch 10 (max stress= $\left.2.437 \mathrm{e}^{\wedge} 007\right)\left(\mathrm{N} / \mathrm{m}^{\wedge} 2\right)$

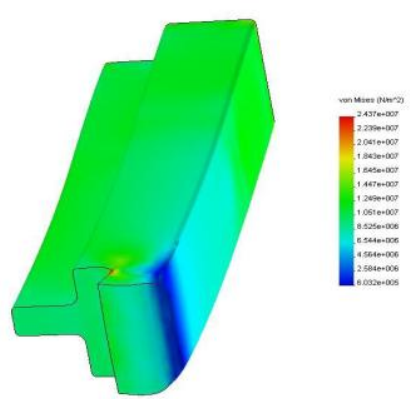

$k^{x}$

Fig 4.2.10 forming punch 10
4.2.11 Analysis of Forming Punch 11 (max stress= $\left.1.209 \mathrm{e}^{\wedge} 008\right)\left(\mathrm{N} / \mathrm{m}^{\wedge} 2\right)$
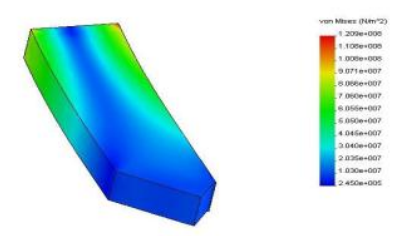

$v^{2}$

Fig 4.2.11) forming punch11

4.2.12 Analysis of Forming Punch 12 (max stress= $\left.1.098 \mathrm{e}^{\wedge} 008\right)\left(\mathrm{N} / \mathrm{m}^{\wedge} 2\right)$
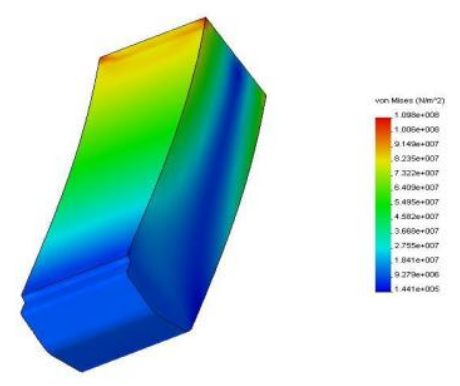

Fig 4.2.12 forming punch12

4.2.13) Analysis of Forming Punch 13 (max stress= $\left.8.236 \mathrm{e}^{\wedge} 007\right)\left(\mathrm{N} / \mathrm{m}^{\wedge} 2\right)$

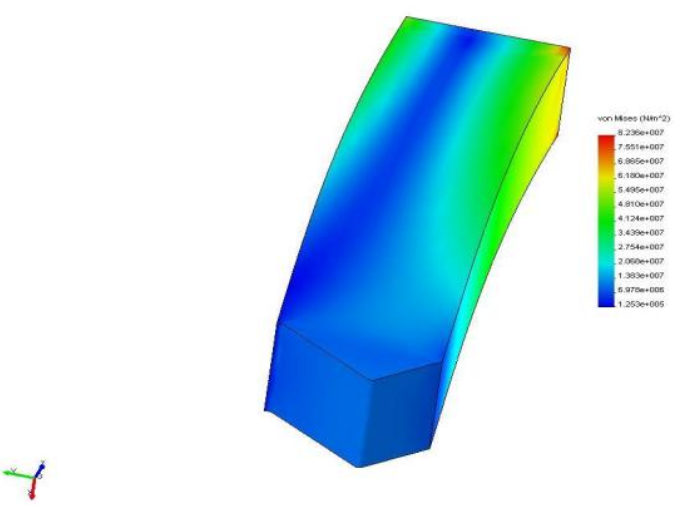

Fig 4.2.13 forming punch13 


\subsubsection{Analysis of Forming Punch 14 (max stress= $\left.8.385 \mathrm{e}^{\wedge} 007\right)\left(\mathrm{N} / \mathrm{m}^{\wedge} 2\right)$}

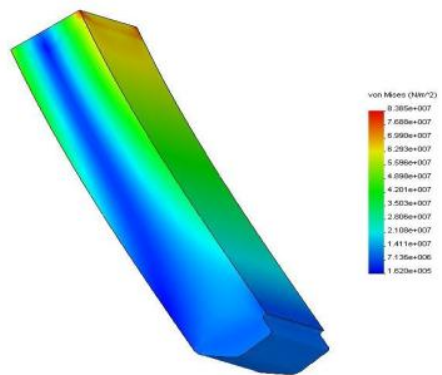

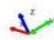

Fig 4.2.14 forming punch14

\subsubsection{Analysis of Forming Punch 15 (max stress=} $\left.1.091 \mathrm{e}^{\wedge} 008\right)\left(\mathrm{N} / \mathrm{m}^{\wedge} 2\right)$
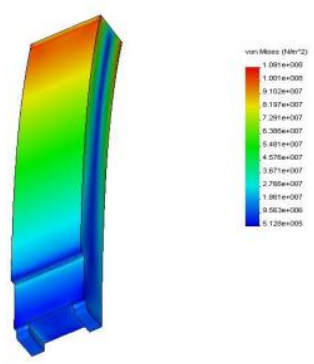

Fig 4.2.15 forming punch15

\subsubsection{6) Analysis of Forming Punch 16 (max stress=} $\left.1.993 \mathrm{e}^{\wedge} 007\right)\left(\mathrm{N} / \mathrm{m}^{\wedge} 2\right)$

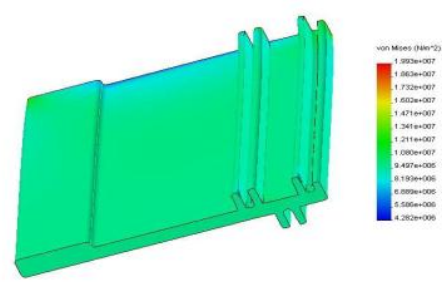

Fig 4.2.16 forming punch16

\subsubsection{Analysis of Forming Punch 17 (max stress= $\left.2.393 \mathrm{e}^{\wedge} 007\right)\left(\mathrm{N} / \mathrm{m}^{\wedge} 2\right)$}

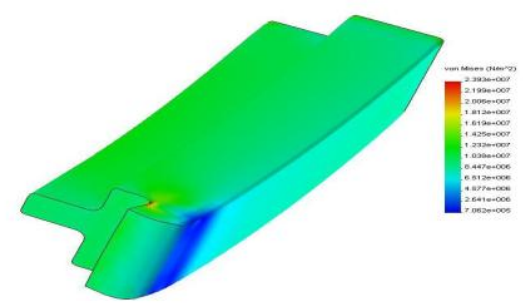

Fig 4.2.17 forming punch17

\section{CALCULATION AND DISCUSSIONS}

\subsection{Production Rate}

NOTE-the following calculations were done based on the information's collected from MSM HYDROPNEUMATIC INDUSTRY and only for 3 operation and by using only one tool for each operation. By referring MSM HYDROPNEUMATIC INDUSTRY work study following information were noted down.

\begin{tabular}{|l|l|l|}
\hline OPERATION & $\begin{array}{l}\text { OPERATION } \\
\text { TIME }(\mathrm{min} / \mathrm{pc})\end{array}$ & $\begin{array}{l}\text { MTRL HANDLING } \\
\text { TIME }(\mathrm{min} / \mathrm{pc})\end{array}$ \\
\hline Piercing & 0.003 & 0.016 \\
\hline Blanking & 0.003 & 0.016 \\
\hline Bending & 0.005 & 0.033 \\
\hline
\end{tabular}

\subsubsection{Cycle Time}

Cycle time can be calculated by using the equation

$$
\text { Tc= To }+ \text { Th+Tth------(1) }
$$

Where $\mathrm{Tc}=$ cycle time, $\mathrm{To}=$ operation time, $\mathrm{Th}=\mathrm{mtrl}$ handling time, $\mathrm{Tth}=$ tool handling time.

\subsubsection{For Normal Die}

Since in normal die tool is already arranged that tool handling time becomes zero. That $\mathrm{Tth}=0$

Therefore equation (1) becomes $\mathbf{T c}=\mathbf{T o}+\mathbf{T h}$

\subsubsection{For Progressive Die}

Since in progressive die material handling is made automatic so it is neglected. That is $\mathrm{Tth}=\mathrm{Th}=0$. Therefore equation (1) becomes $\mathbf{T c}=\mathbf{T o}$ 
Calculating cycle time for both the dies results are as followed.

\subsubsection{Batch Production}

Batch production can be calculated by using the equation $\mathrm{Tb}=\mathrm{T}$ su+Q(Tc)----(2) (min/cycle) where $\mathrm{Tb}=$ batch production time, $\mathrm{Tsu}=$ set up time, $\mathrm{Q}=$ number of parts taken foe each set of operation.

\subsubsection{For Normal Die}

By referring the work study of industry it is found that set up time they taken as $10 \mathrm{sec}$ that $0.166 \mathrm{~min} / \mathrm{pc}$

That is equation (2) becomes $\mathrm{Tb}=0.166+1000(\mathrm{Tc})$

\subsubsection{For Progressive Die}

Since in progressive die set up time is not needed so it is taken as zero. That is $\mathrm{Tsu}=0$

The following result can be plot after calculation.

For normal die batch production time $\mathrm{Tb}=181.166$ (min/cycle)

For progressive die batch production time $\mathrm{Tb}=331.187$ (min/cycle)

\subsubsection{Production Rate}

Production rate can be calculated by using equation

$$
\mathbf{T p}=\mathbf{T b} / \mathbf{Q - - - - ( 3 )}
$$

Where $\mathrm{Tp}=$ production time.

For both the dies equation remains the same

After calculation results will be as followed

For normal die production time $\mathbf{T p}=\mathbf{0 . 1 8 1 1 6}(\mathrm{min} / \mathbf{1 0 0 0 p c})$

For progressive die production time $T p=0.116$ (min/1000pc)

to calculate rate of production rate use the equation

$$
\mathbf{R p}=60 / \mathbf{T p}(\mathrm{min} / \mathrm{hr})-(4)
$$

Equation remains same for both the die

After calculation rate of production

For normal die $\mathbf{R p}=331.187(\mathrm{~min} / \mathrm{hr})$
For progressive die $\mathbf{R p}=871.20(\mathrm{~min} / \mathrm{hr})$

\subsubsection{Net Increase In Production Rate}

BY COMPARING THE RATE OF PRODUCTION THAT IS (871.20-331.187) $\mathrm{Rp}=540.0(\mathrm{~min} / \mathrm{hr})$

\begin{tabular}{|c|c|c|}
\hline OPERATIONS & $\begin{array}{c}\text { NORMAL } \\
\text { DIE }(\mathrm{min} / \mathrm{pc})\end{array}$ & $\begin{array}{c}\text { PROGRESSIVE } \\
\text { DIE }(\mathrm{min} / \mathrm{pc})\end{array}$ \\
\hline Piercing & 0.049 & 0.033 \\
\hline Blanking & 0.049 & 0.033 \\
\hline Bending & 0.083 & 0.05 \\
\hline $\begin{array}{c}\text { Total cycle } \\
\text { time }\end{array}$ & 0.181 & 0.116 \\
\hline
\end{tabular}

\subsection{Results}

By the results of above calculations

\subsubsection{Increase in Production Rate with respect to} Time

\subsubsection{For 1 Hour}

By the above calculations it is shown that for 1 hour rate of production increased by $\mathbf{R p}=\mathbf{5 4 0}(\mathrm{min} / \mathbf{h r})$

\subsubsection{For 1 Batch}

For 1 batch taking 8 hours production rate will be, $\mathrm{Rp}=540 * 8=4320(\mathrm{~min} / \mathrm{batch})$

\subsubsection{For 1 Day}

For 1 day taking 3 batches then the production rate will be, $R p=4320 * 3=12960(\mathrm{~min} /$ day $)$

\subsubsection{For 1 Month}

For 1 month if we consider an average of 30 days production rate will be, $\mathbf{R p}=\mathbf{1 2 9 6 0} * \mathbf{3 0}=\mathbf{3 8 8 , 8 0 0}(\mathbf{m i n} / \mathbf{m o n t h})$

\subsubsection{For 1 Year}

For a year by taking 12 months production rate will be, $R p=388,800 * 12=4,665,600$ (min/annum)

\section{CONCLUSIONS}

6.1 By studying the applications, advantages of progressive dies we can conclude that progressive die can only used for batch and continues production. 
6.2 By studying the types of software's used to analyze these dies I can conclude that stress amount that acts on the tools and the parts of progressive dies.

6.3 By calculating the increase in production rate I concluded the exam statistic benefit of over production by progressive dies.

6.4 By analyzing this die we can conclude progressive die has its initial cost very high but it can bring the revolution in the small scale industries if small scale industries install this die.

6.5 Progressive dies not only increases the production rate but also reduces man power.

6.6 Progressive dies also can bring quality and consistency in bringing quality by continues production.

\section{REFERENCES}

\section{Books Referred}

[1] Vince Adam \& Abraham Askanazi "Building better product with Finite Element Analysis" Onward Press, first edition, 1999.

[2] J.N Reddy "An introduction of finite element method" Tata McGraw Hills, 2ndedition (2001)

\section{Websites}

[1] en.wikipedia.org/wiki/progressive die

[2] en.wikipedia.org/wiki/Finite Element Method 